\title{
An Analysis of Saccadic Eye Movements and Facial Images for Assessing Vigilance Levels During Simulated Driving
}

\author{
Akinori Ueno ${ }^{1}$, Shoyo Tei $^{2}$, Tomohide Nonomura ${ }^{2}$, and Yuichi Inoue ${ }^{3}$ \\ ${ }^{1}$ Department of Electric and Electronic Engineering, School of Engineering, \\ Tokyo Denki University, 2-2 Kanda-Nishiki-cho, \\ Chiyoda-ku, Tokyo 101-8457, Japan \\ ${ }^{2}$ Master's Program of Electronic and Computer Engineering, \\ Graduate School of Science and Engineering, \\ Graduate School of Tokyo Denki University, \\ Ishizaka, Hatoyama, Saitama 350-0394, Japan \\ ${ }^{3}$ Japan Somnology Center, Neuropsychiatric Research Institute, \\ 1-24-10 Yoyogi, Shibuya-ku, Tokyo 151-0053, Japan, \\ ueno@eee.dendai.ac.jp, \{syoyo_t, nonomura\}@ff.f.dendai.ac.jp, \\ inouea.somnology.com
}

\begin{abstract}
The authors analyzed facial video recordings and saccadic eye movements during 1-hour simulated driving in 10 subjects. Mean crosscorrelation coefficient between the visually determined facial sleepiness and the proposed index of saccade (i.e. PV/D) for 9 subjects was -0.56 and the maximum coefficient of inverse cross-correlation was 0.83 . Mean crosscorrelation coefficient for 6 repetitive measurements for another subject was 0.72 , and the maximum was 0.84 . Variation in PV/D preceded that in facial sleepiness in 13 of 15 measurements and syncronized with it in other 2 measurements. From these results, we confirmed a fair potential of the PV/D to detect decline in vigilance levels earlier than facial sleepiness. We also revealed that narrow fluctuations throughout the measurement could lead to low inverse cross-correlation below 0.60 between the two indices. Therefore experimenter should pay attention to designing the experimental procedure to ensure broad fuctuations of the subject's vigilance levels in the measurement.
\end{abstract}

Keywords: doze prevention, saccade, facial sleepiness, advanced safety vehicle.

\section{Introduction}

The increasing number of traffic accidents due to the diminished vigilance level of the driver has become a serious concern all over the world. Drivers with diminished vigilance levels suffer from a marked decline in their abilities of perception, recognition, and vehicle control. Therefore such drivers jeopardize not only their own 
lives but also the others' around them. Accordingly, it is essential to develop a vehicle safety system based on driver's vigilance monitoring.

Several researchers have worked on vigilance monitoring of the drivers with a camera. For instance, Ueno H. et al. proposed a drowsiness detection system based on eye closure metrics [1]. Ji et al. presented a prototype real-time computer vision system for monitoring vigilance of a driver using visual cues of eye tracking, eyelid movement, face pose, and gaze [2]. Smith et al. developed a system for analyzing human driver's visual attention using head and facial features including three-dimensional gaze [3]. All of these studies showed viability and robustness of the system for extracting facial or head features. However, relevancy and validity of their indices of vigilance have not been examined quantitatively at all except in [1]. Even in the article [1], another vigilance index defined by the authors was used for the evaluation, and adequacy of the self-defined index has not been verified objectively at all.

Conceivably, this is because there is no gold standard for estimating vigilance levels. This makes the research for the vigilance monitoring quite difficult. In order to acquire driver's acceptance and broad social acceptance to newly proposed vigilance index, the researchers are desired to explain the mechanism by which the index value are affected in accordance with the change in vigilance level. Since vigilance level is usually defined as an activity level in the brain in staying awake, researchers often seek to explain the mechanism using the activity signal of the brain such as electroencephalogram (EEG). However, even for EEG, any sensitive and widelyaccepted metric for estimating vigilance levels has not been proposed yet. And this makes the research of vigilance monitoring more troublesome.

With the above background in mind, our research group has focused on dynamic characteristics of saccadic eye movement (saccade: SC) as a candidate of vigilance index, and have investigated correlations between the characteristics of SC and other vigilance indices that are quantitatively assessed from physiological, psychological, and psychophysical variables, respectively [4]-[11]. In the articles [4]-[8], spectral powers of EEG at $\mathrm{Fz}, \mathrm{Cz}$, and $\mathrm{Pz}$ during eye tracking tasks were analyzed, and were found to correlate closely with standardized peak velocity, standardized duration, and ratio of peak velocity to duration (PV/D) of SC. In the article [6]-[8], correlations with subjective sleepiness of Kwansei-Gaukin Sleepiness Scale (Japanese translation of Stanford sleepiness scale [12]) and also with the score of self-rated vigilance level were investigated through 24-hour sleep deprivation experiment, and results showed high correlations among the SC parameters and subjective vigilance indices. Additionally, we have showed implications between the SC parameters and a newly devised performance index of visual perception [9]-[11]. In view of our previous results and the fact that $\mathrm{SC}$ is the eye movement most frequently generated in driving, the dynamic characteristics of SC seems promising as a sensitive and reliable index for monitoring driver's vigilance levels.

In order to initiate a feasibility study toward a vehicle safety system based on driver's vigilance monitoring, we have launched validation of the proposed vigilance index (PV/D) during simulated driving. In this paper, we report some preliminary results based on an analysis of facial images and SC associated with vigilance levels. 


\section{Materials and Methods}

\subsection{Subjects}

Nine males, aged 21 to 23 , participated in experiment 1 . Another male aged 21 participated in experiment 2 . All subjects possessed uncorrected visual acuity more than 14/20. They didn't wear corrective lenses during the experiments, nor showed ocular and oculomotor pathology. They were instructed to refrain from drinking beverage containing caffeine and from taking any stimulants on the day of the experiment. Informed consent was obtained from all subjects before the experiment.

\subsection{Apparatus}

A custom-built driving simulator was employed in all experiments (see Fig.1). The simulator outputs rotation angle of steering wheel, acceleration signal of the accelerator pedal and lateral position in the lane. Horizontal and vertical monocular eye movements were measured with an optical eye movement monitor (Takei Scientific Instrument, 2930C). Facial image of the driver was recorded with a camcorder (Sharp, VL-MX1 PRO). Electro-oculogram (EOG) in vertical and horizontal directions was also measured with a bioamplifier (Biopac Systems, EOG100C). In addition, electroencephalogram (EEG) at $\mathrm{Fz}, \mathrm{Cz}, \mathrm{Pz}$, and $\mathrm{Oz}$ of the international 10-20 system, and electromyogram (EMG) of mentalis and masseter muscles were measured in the experiment 1 . All signals except the facial image were sampled at $1 \mathrm{kHz}$ with 16 bit resolution, and then stored in a personal computer using a data recording system (Biopac System, MP-150 system).

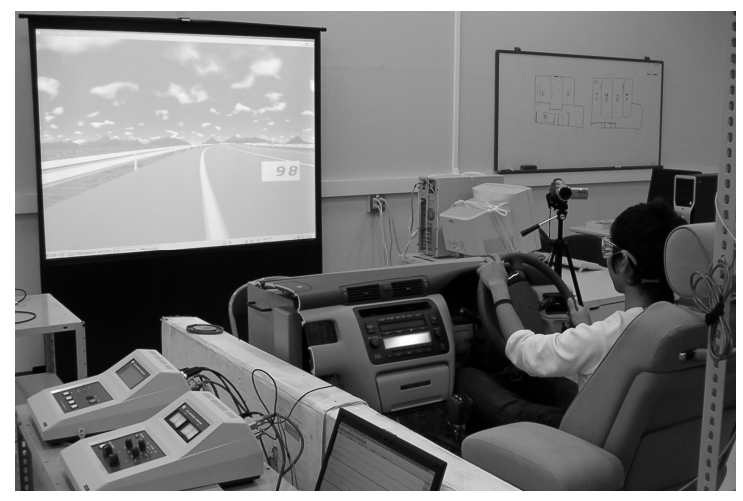

Fig. 1. An image of the custom-built driving simulator employed in this study

\subsection{Experimental Procedure}

In the experiment 1 , the subjects steered the driving simulator for one hour. They instructed to keep a speed at $100 \mathrm{~km} / \mathrm{h}$ in the left lane, but to slow down into $40 \mathrm{~km} / \mathrm{h}$ while a LED cue in the rearview mirror lit up. Lighting up and out of the LED was controlled manually in a random manner by an experimenter. A monotonous and 
repetitive driving course composed of a sine waveform was employed so that the deviation of the car body from the lane could be easily measured. The experiment started at $13: 30$ or $15: 10$.

In the experiment 2, the subject steered the simulator for one hour in the same manner as that described in the experiment 1, but repeated the steering 6 times in different 6 days. The experiments started at 13:30 in the former 3 days and at 15:10 in the latter 3 days.

All subjects had a meal which was served by the experimenter 90 -minute prior to the start of the measurement. Then they practiced the steering more than 10 min until they become accustomed to the simulator. The sensitivity of the eye movement monitor was adjusted prior to start of each experiment. Nine calibration markings which were aligned in a cross shape with a distance of $300 \mathrm{~mm}$ were presented on a front face for the adjustment. The subjects fixated on each marking according to the instruction of the experimenter, while the amplifier gain and offset of eye position signal were inspected and adjusted. Distance between the subject's eye and the center marking was also measured. All experiments were carried out in a darkened room.

\section{Data Analyses}

\subsection{Analysis of Saccade}

Two voltage data obtained from the eye movement monitor were transformed at first to the horizontal and vertical rotation angles using calibration data. Then, the rotation angles were separately differentiated by the two-point central difference algorithm [13] and converted into angular velocity signals. Angular velocity thresholds were set to $\pm 25 \mathrm{deg} / \mathrm{s}$ for detecting SC candidates. In order to discriminate SCs from blinks, an interval threshold was employed. Since the refractory period of SC is known to last more than $50 \mathrm{~ms}$ after the end of each SC motion, SC candidates with an interval less than $50 \mathrm{~ms}$ were regarded as blinks. The reliability of the SC classification algorithm was confirmed in our previous study [14]. Small SCs were discarded because of the limited angular resolution of the monitor. The threshold of amplitude for the small SC was set to $2.0 \mathrm{deg}$. For each selected SC, a synthetic velocity waveform was calculated using the Pythagorean theorem, and then the vigilance index of PV/D was calculated by dividing the peak velocity (PV) by the duration (D) of the SC (see Fig.2). PV/D is an accelerative characteristic having the unit of $\mathrm{deg} / \mathrm{s}^{2}$ and is known to decrease in accordance with decline in vigilance levels in our previous studies [4][11], [15], [16].

PV/D values in each experiment were stamped with time of the SC occurrence in terms of the initiation of the experiment. PV/D values within a period from 0 to $60 \mathrm{~s}$ were pooled by referring to the stamped time and a mean PV/D in the 60-second time window was computed. Then the time window was shifted by $5 \mathrm{~s}$ and corresponding mean PV/D was calculated. This processing was repeated until the end of the window reached the end of the experiment (i.e. $3600 \mathrm{~s}$ ). 


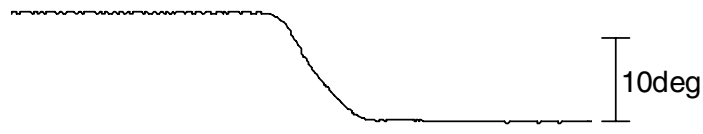

(a) Horizontal rotation angle

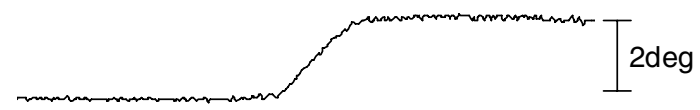

(b) Vertical rotation angle

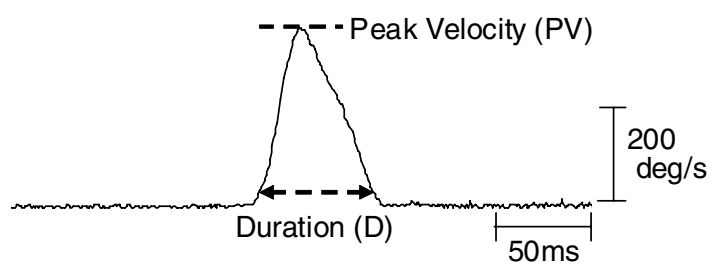

(c) Synthetic velocity

Fig. 2. Records obtained from the eye movement monitor and calculated SC parameters

\subsection{Analysis of Facial Video}

Two trained determiners analyzed the video recording of the subjects' face every $5 \mathrm{~s}$ and rated facial sleepiness (FS) for the video in five levels of 1 to 5 according to the Kitajima's method [15]. Rated two FS for the each 5-second video were averaged, and then moving average was applied to the averaged FS with 60-second time wiondow.

\subsection{Cross-Correlation Analysis}

Cross-correlation between the processed FS and the PV/D was analyzed for each experiment. First 10-minite data were excluded for the analysis to curb improper influence caused by naive rating of FS. Maximum cross-correlation coefficient and the time lag of the FS to the PV/D were computed for each experiment.

\section{Results and Discussion}

\subsection{Results of Experiment 1}

Fig. 3 shows a major example of time-course variations in FS and in PV/D during simulated driving. As can be seen in the figure, the two indices changed quite similarly with time. Particularly reactions to three-time vocal alerts at 2304, 2977 and 


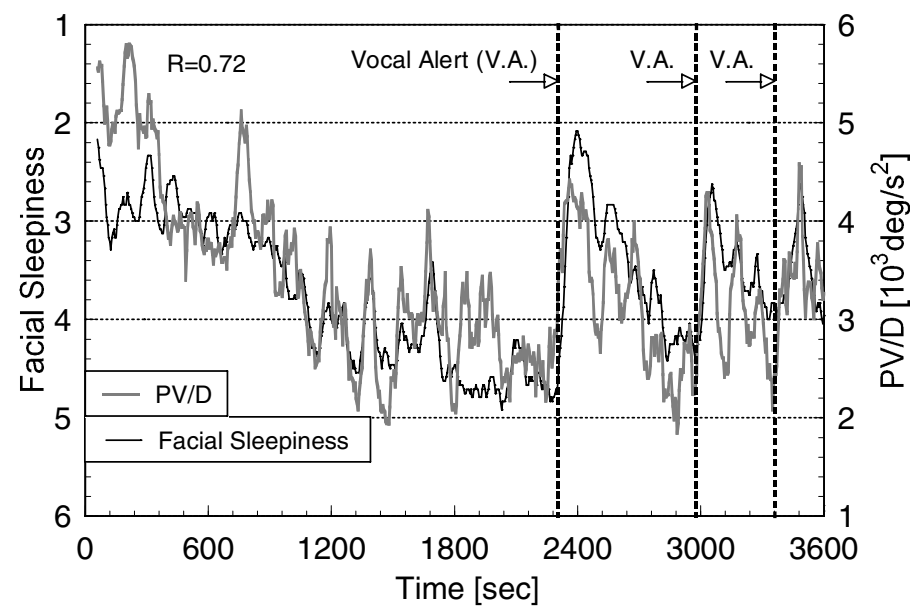

Fig. 3. Time course variations in FS and PV/D during 1-hour simulated driving. Downward direction in vertical axis corresponds to sleepy state and vise versa (Subject B).

Table 1. Cross-correlation coefficient and lag time between FS and PV/D

\begin{tabular}{ccc||ccc}
\hline Subject & $\begin{array}{c}\text { Correlation } \\
\text { Coefficient }\end{array}$ & $\begin{array}{c}\text { Lag Time } \\
\text { [second] }\end{array}$ & $\begin{array}{c}\text { Trial No. } \\
\text { (Subject J) }\end{array}$ & $\begin{array}{c}\text { Correlation } \\
\text { Coefficient }\end{array}$ & $\begin{array}{c}\text { Lag Time } \\
\text { [second] }\end{array}$ \\
\hline A & -0.43 & 15 & $1^{\text {st }}$ & -0.50 & 25 \\
B & -0.72 & 10 & $2^{\text {nd }}$ & -0.72 & 25 \\
C & -0.55 & 15 & $3^{\text {rd }}$ & -0.76 & 15 \\
D & -0.54 & 10 & $4^{\text {th }}$ & -0.84 & 5 \\
E & -0.54 & 10 & $5^{\text {th }}$ & -0.82 & 10 \\
F & -0.71 & 0 & $6^{\text {th }}$ & -0.69 & 10 \\
G & -0.47 & 35 & & & \\
H & -0.23 & 15 & & & \\
I & -0.83 & 0 & & & \\
\hline Ave. & -0.56 & 12.2 & Ave. & -0.72 & 15.0 \\
\hline
\end{tabular}

3360 s were closely synchronized, respectively. As the result of cross-correlation analysis, both variations showed a high correlation of -0.72 and the PV/D preceded 10 $s$ to the FS. Since lag times of FS in Table 1 were greater or equal to 0 in all subjects, PV/D seems having the potential to detect lowering of vigilance level earlier than FS. However, we have to notice that not all of subjects beared high correlations between the indices as in Table 1. One factor has been narrow fluctuation range of the subject's vigilance levels throughout the 1-hour measurement. For detail, we discuss about this in the next subsection.

\subsection{Results of Experiment 2}

As indicated in the right column of Table 1, six repetition of the measurement for one subject revealed wide intraindividual differences (up to 0.34) in cross-correlation 


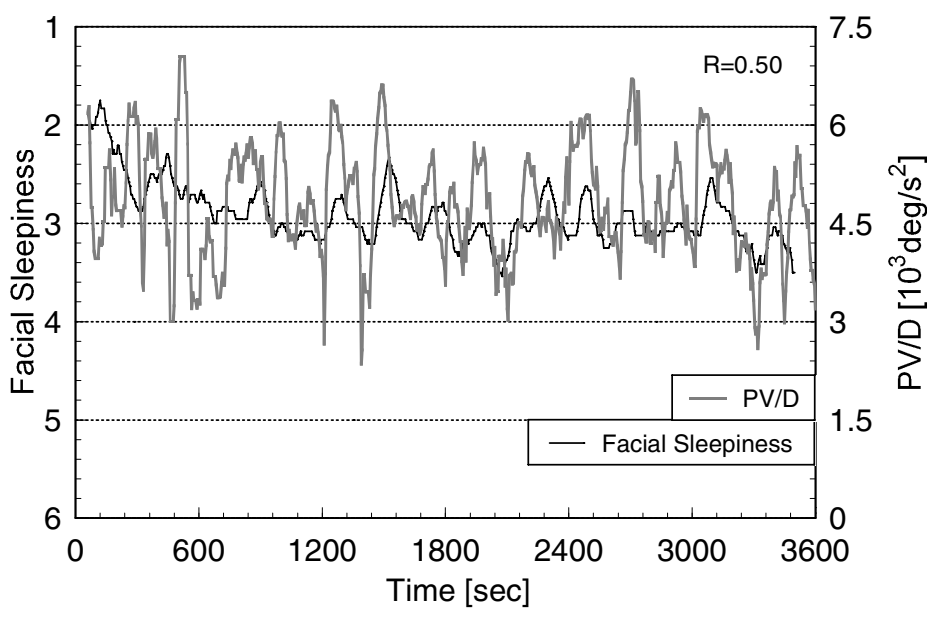

(a) $1^{\text {st }}$ measurement day

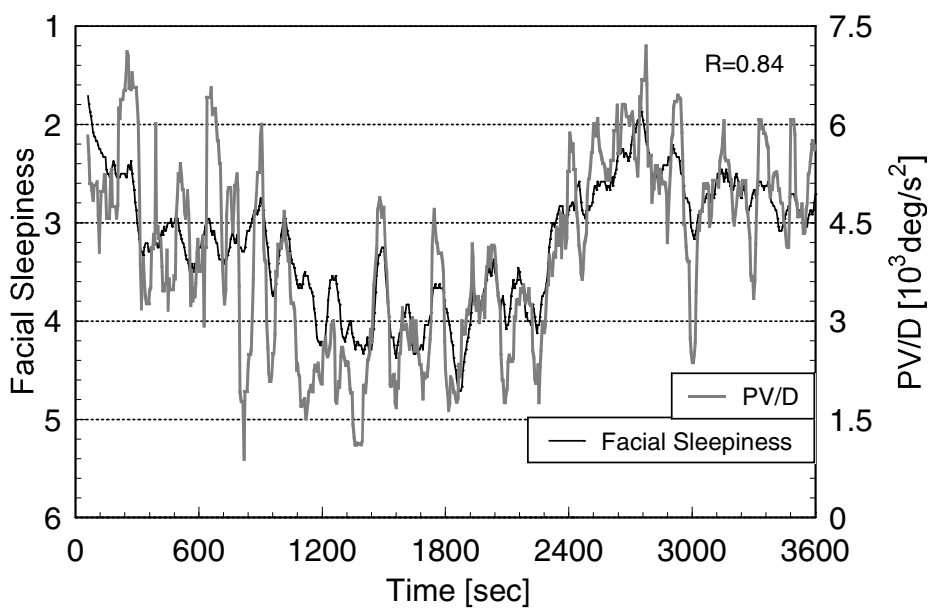

(b) $4^{\text {th }}$ measurement day

Fig. 4. Contrastive results of time-course variations in FS and PV/D in repetitive simulated driving (subject $\mathrm{J}$ ). (a) and (b) correspond to 1st and 4th measurement day, respectively. The lowest and the highest correlation coefficients were obtained in the results of (a) and (b), respectively.

between FS and PV/D. Comparison between time course variations in the indices in Fig.4(a) and (b) suggests that narrow fluctuating range of the subject's vigilance levels throughout the measurement may cause the low cross-correlation. In fact, difference between maximum and minimum FS in the period from 600 to $3600 \mathrm{~s}$ in 
Fig.4(a) was 1.21. By contrast, the difference in Fig.4(b) was 2.88 and more than twice the difference in Fgi.4(a). Furthermore, the summary of the difference for all measurements in Table 2 supports our pypothesis. In Table 2, all measurements in which the difference was less or equal to 2.00 showed low cross-correlation below 0.60. Therefore inter- and intra-individual differences of cross-correlation coefficients between FS and PV/D in Table 1 could attribute mainly to narrow fluctuation of the subject's vigilance levels throughout the measurement. Accordingly, the experienter have to pay more attention to design experimental procedures to ensure broad fuctuations of the subject's vigilance levels in the future.

Table 2. Cross-correlation coefficient between FS and PV/D, and difference between maximum and minimum FS in the period from 600 to $3600 \mathrm{~s}$

\begin{tabular}{ccc||ccc}
\hline Subject & $\begin{array}{c}\text { Correlation } \\
\text { Coefficient }\end{array}$ & $\begin{array}{c}\text { difference } \\
\text { in FS }\end{array}$ & $\begin{array}{c}\text { Trial No. } \\
\text { (Subject J) }\end{array}$ & $\begin{array}{c}\text { Correlation } \\
\text { Coefficient }\end{array}$ & $\begin{array}{c}\text { difference } \\
\text { in FS }\end{array}$ \\
\hline A & -0.43 & 1.58 & $1^{\text {st }}$ & -0.50 & 1.21 \\
B & -0.72 & 2.83 & $2^{\text {nd }}$ & -0.72 & 2.15 \\
C & -0.55 & 1.75 & $3^{\text {rd }}$ & -0.76 & 2.15 \\
D & -0.54 & 1.50 & $4^{\text {th }}$ & -0.84 & 2.88 \\
E & -0.54 & 2.42 & $5^{\text {th }}$ & -0.82 & 2.25 \\
F & -0.71 & 3.08 & $6^{\text {th }}$ & -0.69 & 2.25 \\
G & -0.47 & 2.00 & & & \\
H & -0.23 & 2.75 & & & \\
I & -0.83 & 3.83 & & & \\
\hline
\end{tabular}

\section{Conclusion and Future Issues}

The authors analyzed facial video recordings and saccadic eye movements during 1hour simulated driving in 10 subjects. Mean cross-correlation coefficient between the visually determined facial sleepiness (FS) and the proposed index of saccade (PV/D) for 9 subjects was -0.56 and the maximum coefficient of inverse cross-correlation was 0.83. Mean cross-correlation coefficient for 6 repetitive measurements for another subject was -0.72 , and the maximum was 0.84 . Valiation in PV/D preceded that in FS in 13 of 15 measurements and syncronized with it in other 2 measurements.

From these results, we confirmed a fair potential of the PV/D to detect decline in vigilance levels earlier than FS. We also revealed that narrow fluctuations throughout the measurement could lead to low inverse cross-correlations below 0.60 between the indices. Therefore experimenter should pay attention to designing the experimental procedure to ensure broad fuctuations of the subject's vigilance levels in the experiment.

Future issues to be addressed are as follows:

- reliability analysis of FS in view of concordance rate between the two determiner of the facial sleepiness,

- repeatability investigation of the voltage-degree calibration for eye movement monitoring, 
- analysis of driving performance during the measurment,

- analysis of simultaneously measured EEGs.

\section{Acknowledgement}

This work was advanced as a part of collaborative project with DENSO Corporation.

\section{References}

1. Ueno, H., Kaneda, M., Tsukino, M.: Development of Drowsy Detection System. In: Vehicle Navigation \& Information Systems, pp. 15-20 (1994)

2. Ji, Q., Yang, X.: Real Time Visual Cues Extraction for Monitoring Driver Vigilance. In: Schiele, B., Sagerer, G. (eds.) ICVS 2001. LNCS, vol. 2095, pp. 107-124. Springer, Heidelberg (2001)

3. Smith, P., Shah, M., Lobo, N.V.: Determining Driver Visual Attention with One Camera. IEEE Trans. ITS 4, 205-218 (2003)

4. Ueno, A., Ota, Y., Takase, M., Minamitani, H.: Relationship between Vigilance Levels and Characteristics of Saccadic Eye Movement. In: 17th Ann. Int. Conf. IEEE EMBS, pp. 572-573 (1995)

5. Ueno, A., Ota, Y., Takase, M., Minamitani, H.: Characteristics of Visually Triggered and Internally Guided Saccade Depending on Vigilance States. IEICE Trans. Information and Systems PT 2 J81-D-II, 1411-1420 (1998) (in Japanese)

6. Ueno, A., Ota, Y., Takase, M., Minamitani, H.: Parametric Analysis of Saccadic Eye Movement Depending on Vigilance States. In: 18th Ann. Int. Conf. IEEE EMBS, pp. 319 320 (1996)

7. Ueno, A., Hashimoto, H., Takase, M., Minamitani, H.: Diurnal Variation in Vertical Saccade Dynamics. In: 4th Asia-Pacific Conf. Medical and Biological Engineering, p. 373 (1999)

8. Ueno, A., Tateyama, T., Takase, M., Minamitani, H.: Dynamics of Saccadic Eye Movement Depending on Diurnal Variation in Human Alertness. System and Computers in Japan 33, 95-103 (2002)

9. Ueno, A., Uchikawa, Y.: Relation between Human Alertness, Velocity Wave Profile of Saccade, and Performance of Visual Activities. In: 26th Ann. Int. Conf. IEEE EMBS, pp. 933-935 (2004)

10. Ueno, A., Sakamoto, S., Uchikawa, Y.: Relation between Dynamics of Saccade and Bit Rate for Visual Perception during Numerical Targets Comparing. IEICE Trans. Information and Systems PT 2 J87-D-II, 2062-2070 (2004) (in Japanese)

11. Ueno, A., Uchikawa, Y.: An Approach to Quantification of Human Alertness Using Dynamics of Saccadic Eye Movement: For an Application to Human Adaptive Mechatronics. In: 8th Int. Conf. Mechatronics. Technol., pp. 563-568 (2004)

12. Herscovitch, J., Broughton, R.: Sensitivity of the Stanford Sleepiness Scale to the Effects of Cumulative Partial Sleep Deprivation and Recovery Oversleeping. Sleep 4, 83-92 (1981)

13. Bahill, A.T., Kallman, J.S., Lieberman, J.E.: Frequency limitations of the two-point central difference differentiation algorithm. Biol. Cybern. 45, 1-4 (1982) 
14. Komatsu, Y., Ueno, A., Hoshino, H.: Real-time discrimination of saccade and eye blink from output signal of eye movement monitor. In: 19th Ann. Conf. The Society of Life Support Technology, p. 54 (2003) (in Japanese)

15. Ueno, A., Kokubun, S., Uchikawa, Y.: A Prototype Real-Time System for Assessing Vigilance Levels and for Alerting the Subject with Sound Stimulation. Int. J. Assist. Robotics and Mechatronics 8, 19-27 (2007)

16. Kokubun, S., Ueno, A., Uchikawa, Y.: Influence of Sound Pressure Level of Beep Stimulation on Arousal Effect: Quantitative Evaluation Based on Analysis of Saccade and Electroencephalogram. Trans. SICE 44, 871-877 (2008) (in Japanese)

17. Kitajima, H., Numata, N., Yamamoto, K., Goi, Y.: Prediction of Automobile Driver Sleepiness: 1st Report, Rating of Sleepiness Based on Facial Expression and Examination of Effective Predictor Indexes of Sleepiness. Trans. JSME Series C 63, 3059-3066 (1997) (in Japanese) 